\title{
PATTERN FORMATION FOR A RATIO-DEPENDENT PREDATOR-PREY MODEL WITH CROSS DIFFUSION
}

\author{
M. SAMBATH ${ }^{\dagger}$ AND K. BALACHANDRAN
}

Department of Mathematics, Bharathiar University, Coimbatore 641 046, India.

E-mail address: sambathbu2010@gmail.com, kb.maths.bu@gmail.com

\begin{abstract}
In this work, we analyze the spatial patterns of a predator-prey system with cross diffusion. First we get the critical lines of Hopf and Turing bifurcations in a spatial domain by using mathematical theory. More specifically, the exact Turing region is given in a two parameter space. Our results reveal that cross diffusion can induce stationary patterns which may be useful in understanding the dynamics of the real ecosystems better.
\end{abstract}

\section{INTRODUCTION}

Beginning from the last century, numerous biological models have been received extensive attention. In particular, the predator-prey models have been of great interest to both applied mathematicians and ecologists. The first model to describe the size (density) dynamics of two populations interacting as a predator-prey system was developed independently by Alfred Lotka (1925) and Vito Volterra (1931). Since the classical Lotka-Volterra models suffer from some unavoidable limitations in describing precisely many realistic phenomena in biology, in some cases, they should make way to some more sophisticated models from both mathematical and biological points of view.

All the beings, including different kinds of populations, live in a spatial world and it is a natural phenomenon that a substance goes from high-density regions to low-density regions. As a result, more and more scholars use spatial model to study the interaction of the prey and predator [12]. Recently there has been considerable interest to investigate the stability behavior of a system of interacting populations by taking into account the effect of self as well as cross diffusion [14]. The term self diffusion implies the movement of individuals from a higher to lower concentration region. Cross diffusion expresses the population fluxes of one species due to the presence of the other species. The value of the cross diffusion coefficient may be positive, negative or zero. The positive cross diffusion coefficient denotes the movement of the species in the direction of lower concentration of another species and negative cross diffusion

Received by the editors September 27 2012; Accepted December 72012.

2010 Mathematics Subject Classification. 92D25, 70K50, 35B36.

Key words and phrases. Cross diffusion, Turing bifurcation, Predator-prey model, Pattern formation.

${ }^{\dagger}$ Corresponding author. 
coefficient denotes that one species tends to diffuse in the direction of higher concentration of another species [8].

In 1952, A.M. Turing first proposed the reaction-diffusion theory for pattern formation in his seminal work on the chemical basis of morphogenesis [21]. A fact in which a non-linear system is asymptotically stable in the absence of self and cross diffusions but unstable in the presence of self and cross diffusions is known as Turing instability. This concept has been playing significant roles in theoretical ecology, embryology and other branches of science. Similarly structured systems of ordinary differential equations govern the spatiotemporal dynamics of ecological population models, yet most of the simple models predict spatially homogeneous population distributions. One notable exception to this rule was demonstrated by Bartumeus et al. $[1,3]$ who reported that intra-predator interaction, or interference, may facilitate spatial pattern formation in a variation of the DeAngelis model [7, 15].

It has been observed that the spatially extended reaction-diffusion models for prey-predator interaction and various kinds of spatial patterns have been reported (see $[4,10,18,19])$. A limited number of papers has appeared on resulting patterns exhibited by spatiotemporal preypredator model with ratio-dependent functional response. Banerjee [2] performed the linear stability analysis for a diffusive predator-prey model with ratio-dependent functional response for the predator and reported the diffusion driven instability behavior and resulting Turing structures with heterogeneous environment. Earlier Fan and Li [9] studied the global asymptotic stability of the unique positive constant equilibrium solution for the same type of ratiodependent prey-predator system with diffusion term. Recently Liu and Jin [13] analyze spatial pattern formation of a ratio-dependent predator-prey system with self diffusion. To the author's knowledge, the cross diffusion pattern generated by a ratio-dependent prey-predator model has not yet been reported by any researcher till date. The main objective of the present paper is to consider a two species prey-predator model with ratio-dependent functional response distributed over two-dimensional state space and establishes pattern formation for certain choices of initial population distribution.

Our paper is organized as follows. In Section 2, we analyze the predator-prey model with cross diffusion and derive the mathematical expressions for the Hopf and Turing bifurcation critical lines. In section 3, we present the result of pattern formation via numerical simulation. Finally we present some conclusion and discussion in section 4.

\section{THE MODEL AND HOPF BIFURCATION ANALYSIS}

The dynamics of ratio-dependent predator-prey system is governed by the following first order non-linear ordinary differential equations

$$
\left\{\begin{array}{l}
\dot{u}(t)=r u\left(1-\frac{u}{K}\right)-\frac{\alpha u v}{(v+\alpha \beta u)}, \\
\dot{v}(t)=v\left(-\gamma+\frac{e \alpha u}{(v+\alpha \beta u)}\right), \\
u(t=0)=u_{0}>0, \quad v(t=0)=v_{0}>0,
\end{array}\right.
$$


where $u$ and $v$ are prey and predators, respectively. $r$ represents intrinsic growth rate of the prey and carrying capacity $K$ in the absence of predation, $e$ conversion efficiency, $\alpha$ total attack rate for predator, $\beta$ the handling time and $\gamma$ death rate of the predator. The predator consumes the prey with the functional response known as Michaelis-Menten-Holling type functional response [11].

In order to minimize the number of parameters involved in the model, it is extremely useful to write the system in non-dimensionalized form. Thus we take

$$
u \mapsto u / K, \quad v \mapsto v / K \alpha \beta \text { and consider the dimensionless time } t \mapsto r t
$$

and then we arrive at the equations of the following form,

$$
\left\{\begin{array}{l}
\dot{u}(t)=u(1-u)-\frac{a u v}{(u+v)}, \\
\dot{v}(t)=c\left(-v+\frac{b u v}{(u+v)}\right),
\end{array}\right.
$$

where $a=\alpha / r, b=e / \beta \gamma$ and $c=\gamma / r$.

Thus the model with cross diffusion becomes

$$
\left\{\begin{array}{l}
u_{t}=d_{11} \Delta u+d_{12} \Delta v+u(1-u)-\frac{a u v}{(u+v)}, \\
v_{t}=d_{21} \Delta u+d_{22} \Delta v+c\left(-v+\frac{b u v}{(u+v)}\right) .
\end{array}\right.
$$

In the above, $\Delta$ is the Laplacian operator in two-dimensional space, $d_{11}, d_{22}$ are self diffusion coefficients of prey and predator, $d_{12}, d_{21}$ are the cross diffusion coefficients of prey and predator respectively.

The model (2.3) is analyzed with the initial populations $u(0)>0, v(0)>0$.

We also assume that no external input is imposed from outside. Hence the boundary condition are taken as

$$
\left.\frac{\partial u}{\partial \nu}\right|_{(x, y)}=\left.\frac{\partial v}{\partial \nu}\right|_{(x, y)}=0, \quad(x, y) \in \partial \Omega,
$$

where $\nu$ is the outward unit normal vector on $\partial \Omega$ and $\Omega$ is the two-dimensional spatial domain.

We are interested mostly in(from biological point of view) positive equilibrium point $E^{*}=$ $\left(u^{*}, v^{*}\right)$, which corresponds to co-existence of prey and predator and is given by

$$
u^{*}=\frac{a+b-a b}{b}, \quad v^{*}=u^{*}(b-1) .
$$

It is easy to obtain the condition for ensuring that $u^{*}$ and $v^{*}$ are positive as $1<b<a /(a-$ $1), a>1$.

We are interested in studying the stability behavior of the positive equilibrium point $E^{*}$. The Jacobian evaluated at the coexistence equilibrium $E^{*}=\left(u^{*}, v^{*}\right)$ is

$$
J=\left(\begin{array}{ll}
a_{11} & a_{12} \\
a_{21} & a_{22}
\end{array}\right) .
$$


where

$$
a_{11}=a-\frac{a}{b^{2}}-1, \quad a_{12}=-\frac{a}{b^{2}}, \quad a_{21}=\frac{(b-1)^{2} c}{b}, \quad a_{22}=\left(\frac{1}{b}-1\right) c .
$$

We linearize the predator-prey system (2.3) around the spatially homogeneous fixed point $\left(u^{*}, v^{*}\right)$ as follows:

$$
\left(\begin{array}{l}
u(\vec{\eta}, t) \\
v(\vec{\eta}, t)
\end{array}\right)=\left(\begin{array}{l}
u^{*} \\
v^{*}
\end{array}\right)+\left(\begin{array}{c}
\hat{u}(\eta, t) \\
\hat{v}(\eta, t)
\end{array}\right),
$$

where $|\hat{u}(\eta, t)|<<u^{*}, \quad|\hat{v}(\eta, t)|<<v^{*}$ and $\vec{\eta}$ is in two-dimensional space.

By setting

$$
\left(\begin{array}{c}
u(\vec{\eta}, t) \\
v(\vec{\eta}, t)
\end{array}\right)=\left(\begin{array}{c}
u_{0} e^{\lambda t} e^{i \vec{k}, \hat{\eta}} \\
v_{0} e^{\lambda t} e^{i \vec{k}, \hat{\eta}}
\end{array}\right),
$$

we obtain the characteristic equation

$$
\left|J-\lambda I-k^{2} D\right|=0
$$

where

$$
D=\left(\begin{array}{ll}
d_{11} & d_{12} \\
d_{21} & d_{22}
\end{array}\right)
$$

Now we obtain the characteristic polynomial from (2.8) as follows

$$
\lambda^{2}+T_{k} \lambda+D_{k}=0
$$

where

$$
\begin{aligned}
& T_{k}=\left(d_{11}+d_{22}\right) k^{2}-\left(a_{11}+a_{22}\right), \\
& D_{k}=\left(d_{11} d_{22}-d_{12} d_{21}\right) k^{4}-\left(d_{11} a_{22}+d_{22} a_{11}-d_{12} a_{21}-d_{21} a_{12}\right) k^{2}+\left(a_{11} a_{22}-a_{12} a_{21}\right) .
\end{aligned}
$$

The roots of (2.10) are given by

$$
\lambda_{k}=\frac{-T_{k} \pm \sqrt{T_{k}^{2}-4 D_{k}}}{2} .
$$

The onset of Hopf instability corresponds to the case when a pair of imaginary eigenvalues cross the real axis from the negative to positive side and this situation occurs only when the diffusion vanishes. Mathematically speaking, the Hopf bifurcation occurs when

$$
\operatorname{Im}(\lambda(k)) \neq 0, \quad \operatorname{Re}(\lambda(k))=0 \text { at } k=0 .
$$

Then we get the critical value of the Hopf bifurcation parameter $c$, equal to $c_{H}$, where

$$
c_{H}=\frac{a+b^{2}(1-a)}{b(1-b)} .
$$

The positive equilibrium point $\left(u^{*}, v^{*}\right)$ will be unstable if at least one of the roots of (2.10) is positive. By straight forward analysis, we find that $D_{k}$ is a quadratic polynomial with respect to $k^{2}$. Its extremum is a minimum at some $k^{2}[17,20]$. From $D_{k}$, elementary differentiation with respect $k^{2}$ shows that

$$
k_{\min }^{2}=\frac{d_{11} a_{22}+d_{22} a_{11}-d_{12} a_{21}-d_{21} a_{12}}{2 \operatorname{det}(D)} .
$$


At the critical point, we have $D_{k}=0$ when $k=k_{c r}$ [20]. For fixed kinetics parameters, this define a critical cross diffusion coefficient $d_{12}$ as the root of equation

$$
\left(d_{11} a_{22}+d_{22} a_{11}-d_{12} a_{21}-d_{21} a_{12}\right)^{2}-4 \operatorname{det}(J) \operatorname{det}(D)=0 .
$$

The critical wavenumber $k_{c r}$ is given by

$$
k_{c r}=\sqrt{\frac{\operatorname{det}(J)}{\operatorname{det}(D)}} .
$$

A general linear analysis $[1,5,6]$ shows that the necessary conditions for yielding Turing patterns are given by

$$
\begin{aligned}
& a_{11}+a_{22}<0, \\
& a_{11} a_{22}-a_{12} a_{21}>0, \\
& \left(d_{11} a_{22}+d_{22} a_{11}-d_{12} a_{21}-d_{21} a_{12}\right)>0, \\
& \left(d_{11} a_{22}+d_{22} a_{11}-d_{12} a_{21}-d_{21} a_{12}\right)^{2}-4 \operatorname{det}(J) \operatorname{det}(D)>0 .
\end{aligned}
$$

Mathematically speaking, the Turing bifurcation occurs when

$$
\operatorname{Im}(\lambda(k))=0, \quad \operatorname{Re}(\lambda(k))=0 \text { at } k=k_{c r} \neq 0 .
$$

and the wave number $k_{c r}$ is the same as in (2.12). By direct calculation, we obtain the critical value of bifurcation parameter $c$, equal to $c_{T}$, where

$$
\begin{aligned}
c_{T}= & \frac{1}{b^{2}(b-1)^{2}\left(d_{11}+(b-1) d_{12}\right)^{2}} \\
& \left(2 \sqrt{b^{3}(b-1)^{2}(a+b(1-a))\left(a\left(d_{11}-d_{12}\right)+b^{2} d_{12}(a-1)\right)\left(d_{12}+d_{22}(b-1)\right)(\operatorname{det}(D))}\right. \\
& +b(b-1)\left(a\left(d_{11}+(b-1)(2 b+1) d_{12}\right) d_{21}+a(b-1)^{2}\left(d_{12}(1+b)-d_{11}\right) d_{22}\right. \\
& \left.\left.+b^{2}\left(-2 d_{12} d_{21}+\left(d_{11}+d_{12}(1-b)\right) d_{22}\right)\right)\right),
\end{aligned}
$$

where $\operatorname{det}(J)=a_{11} a_{22}-a_{12} a_{21}$ and $\operatorname{det}(D)=d_{11} d_{22}-d_{12} d_{21}$.

We fix the deterministic model values $a=2.6, b=1.6, d_{11}=0.5, d_{21}=-0.1, d_{22}=10$ and vary $d_{12}$ as a function of $c$ which is the coefficient of the cross diffusion of the prey. Now we discuss the bifurcations represented by these formulas in the parameter space. The bifurcation lines divide the parameter space into three distinct regions (see Fig. $1(A)$ ). The upper part of the displayed parameter space (where it is marked by III) corresponds to systems with homogeneous unconditionally stable equilibria. In region I, both Hopf and Turing bifurcations occur. The equilibria that can be found in the area, marked II, are stable with respect to homogeneous perturbations but lose their stability to homogeneous perturbations of specific wavenumber $k$.

Fig. $1(B)$ shows the Turing space properly. The dispersion relation of the model (2.3) with several values of the one parameter fixed $c=5$. It can be see from Fig. $1(B)$ that when $d_{12}$ increases, the available Turing models $[\operatorname{Re}(\lambda)>0]$ decrease and all available models are weakened.

\section{PATTERN FORMATION}

The dynamical behavior of the spatial predator-prey model cannot be determined by using analytical methods or normal forms. Thus we have to perform numerical simulations by computer. The main aim is to check whether a spatially homogeneous equilibrium is stable, unstable and to which solution to converges. 

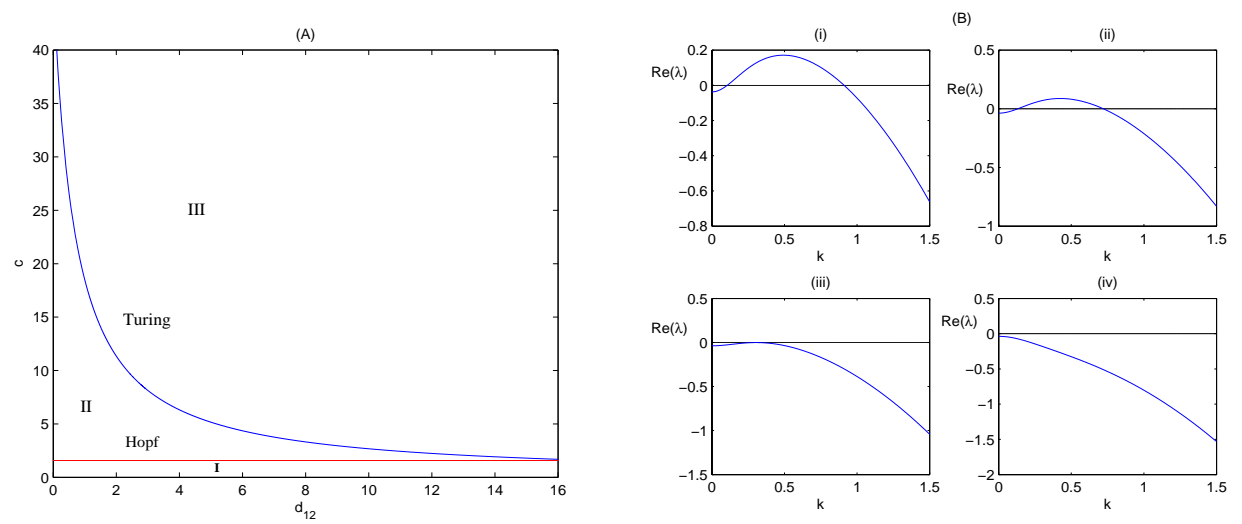

Fig.1 $(A)$ The bifurcation diagram of model (2.3) with the parameter values $a=2.6, b=1.6$, $d_{11}=0.5, d_{21}=-0.1$ and $d_{22}=10$. The red and blue lines correspond to the Hopf $\left(c_{H}\right)$ and Turing $\left(c_{T}\right)$ bifurcation critical lines respectively. The figure shows the Turing space (it is marked by II) with the area bounded by the Turing bifurcation line and the Hopf bifurcation line.

$(B)$ Variation of dispersion relation of the model (2.3) with the parameter values $a=2.6, b=1.6, c=5, d_{11}=0.5, d_{21}=-0.1$ and $d_{22}=10$. (i) $d_{12}=0.5, \quad$ (ii) $d_{12}=1.8$,

(iii) $d_{12}=3.4$ (Turing instability occurs), $(i v) d_{12}=7$ (Turing instability decays).
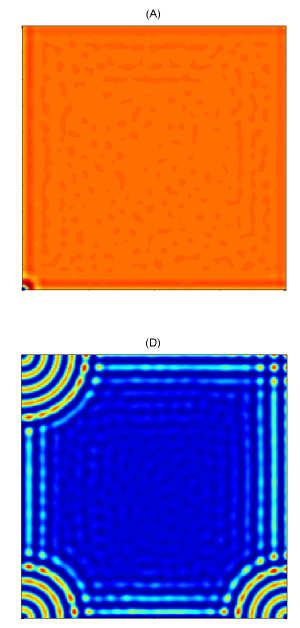
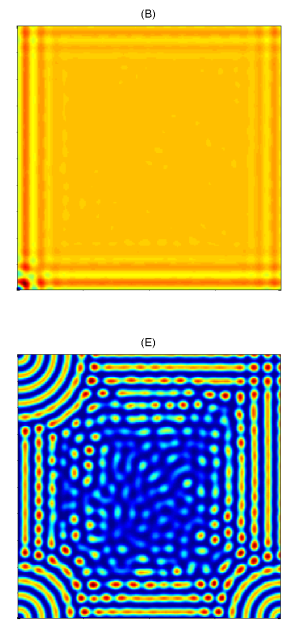
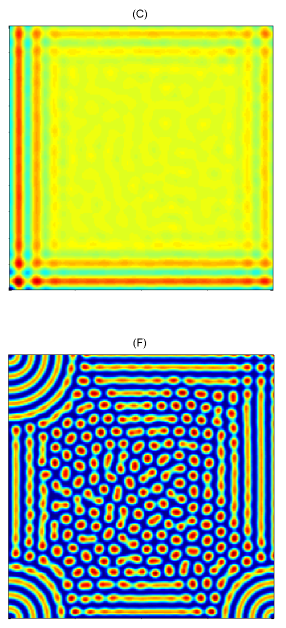

Fig. 2 Snapshots of contour of the time evolution of the prey at different instants with $a=2.6$, $b=1.6, c=5, d_{11}=0.5, d_{21}=-0.1, d_{22}=10$ and $d_{12}=0.5$ and the parameter values in the Turing space. $(A) t=0,(B) t=100,(C) t=250,(D) t=350,(E) t=500,(F) t=1000$.

The problem defined by the reaction-diffusion system in two-dimensional space domain is solved in a discrete domain with $M \times N(M=N=200)$ lattice sites. The spacing in between the lattice points is defined by the lattice constants $\Delta h$. In the discrete system, the Laplacian describing diffusion is calculated using finite difference schemes, that is, the derivatives are approximated by differences over 

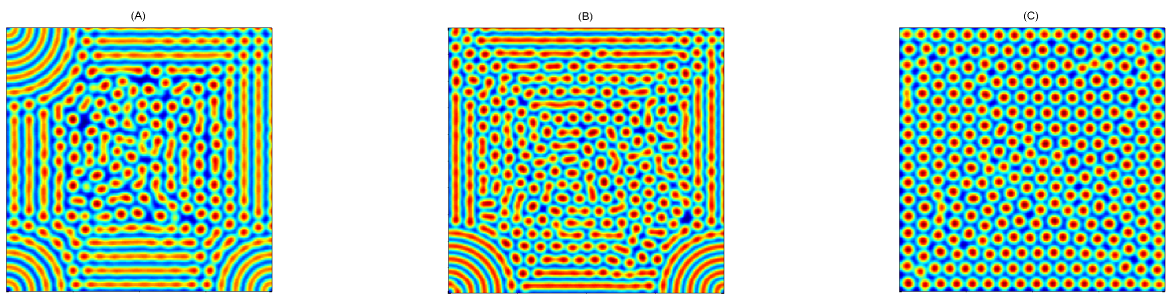

Fig. 3 Snapshots of contour of the prey at $t=1000$ with $a=2.6, b=1.6, c=5, d_{11}=0.5$, $d_{21}=-0.1$ and $d_{22}=10$. $(A) d_{12}=1.5,(B) d_{12}=3,(C) d_{12}=7$. The parameters values are chosen values here in the Turing space.

$\Delta h$. For $\Delta h \rightarrow 0$ the differences approach the derivatives. The time evolution is also discrete, that is, the time goes by steps of $\Delta t$ and it can be solved by using Euler's method. The model (2.3) is solved by numerically approximating the spatial derivatives and an explicit Euler's method for the time integration with a time step size of $\Delta t=0.01$ and space step size $\Delta h=0.1$. All our numerical simulations employ the non-zero initial conditions and the Neumann boundary conditions.

Fig. 2 shows the evolution of the spatial pattern of the prey at $t=0,100,250,350,500$, and 1000, with small random perturbation of the stationary solution $u^{*}$ and $v^{*}$ of the spatially homogeneous system with $d_{12}=0.5$. In this case, one see that for the system (2.3) the random initial distribution leads to formation of irregular patterns. As the time is increased, spotted patterns and some striped patterns prevail over the whole domain finally and the dynamics of the system does not undergo any further change.

Fig. $3(A)$ show the evolution of the spatial pattern of the prey at $t=1000$ with small random perturbation of the stationary solution $u^{*}$ and $v^{*}$ of the spatially homogeneous system with $d_{12}=1.5$. One can see from this figure that the strips-spots mixture patterns prevail in the whole domain.

Fig. $3(B)$ show the evolution of the spatial pattern of the prey at $t=1000$ with small random perturbation of the stationary solution $u^{*}$ and $v^{*}$ of the spatially homogeneous system with $d_{12}=3$. One can see from this figure that the spots and labyrinth mixture patterns prevail in the whole domain.

As $d_{12}$ increases to 7 , we show the spatial pattern of prey at $t=1000$ in Fig. $3(C)$. We see from the figure, the spotted patterns of spatial over the whole domain.

\section{CONCLUSION}

This article presents the pattern formation of a spatially extended ratio-dependent predator-prey model in two-dimensional space. Based on both mathematical analysis and numerical simulations, we find that its spatial pattern includes spotted patterns.

From the biological point of view, our results have some clear meaning. There has been a growing understanding, during the past years, regarding the dynamics of real ecosystems. It is important to reveal the different spatial dynamical regimes arising as a result of perturbation of the system parameters $[10,16]$. The numerical simulation results indicate that the effect of the cross diffusion for pattern formation is remarkable. More specifically, as the value of predator cross diffusion coefficient $d_{12}$ is increased, the spotted stripe-like and labyrinth patterns emerge (see Fig 2,3). This enriches the dynamics of the effect of the cross diffusion of the predator-prey model. 


\section{REFERENCES}

[1] D. Alonso, F. Bartumeus and J. Catalan, Mutual interference between predators can give rise to Turing spatial patterns, Ecology. 83 (2002), 28-34.

[2] M. Banerjee, Self-replication of spatial patterns in a ratio-dependent predator-prey model, Math. Comput. Modelling. 51 (2010), 44-52.

[3] F. Bartumeus, D. Alonso and J. Catalan, Self-organized spatial structures in a ratio-dependent predator-prey model, Physica A. 295 (2001), 53-57.

[4] D.L. Benson, J. Sherratt and P.K. Maini, Diffusion driven instability in an inhomogeneous domain, Bull.Math.Biol. 55 (1993), 365-384.

[5] J.M. Chung and E. Peacock-Lpez, Bifurcation diagrams and Turing patterns in a chemical self-replicating reaction-diffusion system with cross diffusion, J. Chem. Phys. 127 (2007), 174903.

[6] J.M. Chung and E. Peacock-Lpez, Cross-diffusion in the templator model of chemical self-replication, Phys. Let. A. 371 (2007), 41-47.

[7] D.L. DeAngelis, R.L. Goldstein and R.V. O’Neill, A model for trophic interaction, Ecology. 56 (1975), 881892.

[8] B. Dubey , B. Das and J. Hussain, A predator - prey interaction model with self and cross-diffusion, Ecol. Model. 141 (2001), 67-76.

[9] Y.H. Fan and W.T. Li, Global asymptotic stability of a ratio-dependent predator prey system with diffusion, J.Comput.Appl.Math. 188 (2006), 205-227.

[10] M.R. Garvie, Finite-Difference schemes for reaction diffusion equations modelling predator prey interactions in MATLAB, Bull.Math.Biol. 69 (2007), 931-956.

[11] C. Jost, O. Arino and R. Arditi, About deterministic extinction in ratio-dependent predator-prey model, Bull.Math.Biol. 61 (1999), 19-32.

[12] S.A. Levin, T.M. Powell and J.H. Steele, Patch Dynamics, Lecture Notes in Biomathematics, Springer-Verlag, Berlin, 1993.

[13] P.P. Liu and Z. Jin, Pattern formation of a predator-prey model, Nonlinear Anal. Hybrid Syst. 3 (2009), 177183.

[14] Y. Lou and W. M. Ni, Diffusion vs cross-diffusion: An elliptic approach, J. Differential Equations. 154 (1999), 157-190.

[15] E.A. McGehee and E. Peacock-Lpez, Turing patterns in a modified Lotka Volterra model, Phys. Lett. A. 342 (2005), 90-98.

[16] A. Morozov, S.Petrovskii and B.L. Li, Bifurcations and chaos in a predator-prey system with the allee effect, Proc. R. Soc. Lond. B 271 (2004), 1407-1414.

[17] J.D. Murray, Mathematical Biology, Springer-Verlag, Berlin, 1993.

[18] M. Sambath, S. Gnanavel and K. Balachandran, Stability and Hopf bifurcation of a diffusive predator-prey model with predator saturation and competition, Anal. Appl. 1-18 (2012) DOI: 10.1080/00036811.2012.742185

[19] J.A. Sherratt, Turing bifurcations with a temporally varying diffusion coefficient, J.Math.Biol. 33 (1995), 295308.

[20] X.K. Sun, H.F. Huo and H. Xiang, Bifurcation and stability analysis in predator-prey model with a stagestructure for predator, Nonlinear Dyn. 58 (2009), 497-513.

[21] A.M. Turing, The chemical basis of morphogenesis, Philos. Trans. R. Soc. Lond. Ser. B: Biol. Sci. 237 (1952), 37-72. 